\title{
Special Section Guest Editorial: Image and Video Compression Using Deep Neural Networks
}

\author{
Ofer Hadar ${ }^{a}$ and Touradj Ebrahimi ${ }^{b}$

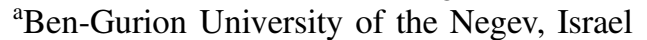 \\ ${ }^{\mathrm{b}}$ Swiss Federal Institute of Technology, Lausanne, Switzerland
}

Multiple research efforts are performed to harness the predictive and classification powers of deep neural networks (DNN) to improve video and image compression algorithms. The Special Section on Image and Video Compression Using Deep Neural Networks presents five recent papers that leverage various deep neural network architectures to improve video and image compression.

In the field of hyperspectral images (HSIs), Chong et al. propose an end-to-end optimization in the form of joint spectral-spatial compression and reconstruction 3D convolutional autoencoder, which improves the spectral angle mapper (SAM), peak signal-to-noise ratio (PSNR), and structural similarity index measurement (SSIM) of the reconstructed HSIs by $20.8 \%-33.1 \%$, $0.9 \%-11.5 \%$, and $0.5 \%-2.2 \%$, respectively, relative to competitive methods.

In the field of compressed sensing (CS), where, under certain conditions, signals can be sampled at sub-Nyquist rates while still enabling exact reconstruction from linear projections of the signal, Pan et al. propose a novel block-based CS (BCS) image reconstruction framework via a deep learning network with smoothed projected Landweber (SPL). A fully connected network performs both BCS linear sensing and non-linear reconstruction stages, and SPL removes the blocking artifacts due to incorporate Wiener filtering into projected Landweber (PL) method at each iteration. At $10 \%$ sensing rate, the structural similarity maximum (minimum) gain reaches 0.098 (0.021). In the same field, Cohen et al. propose a one-dimensional convolutional neural network $(\mathrm{CNN})$ for spectral classification of CS images that is applied directly on the compressive measurements. They show a reduction of captured data by an order of magnitude without significant loss in the classification performance.

A CNN compression artifact reduction in images is proposed by X. Zhang et al., whereas a novel progressive multi-scale attention network is used and further enhanced with a generative adversarial network (GAN) to improve image quality. The proposed network architecture removes compression artifacts such as blocking, blurring or ringing effects, which are inevitable when compression rate is high.

The challenge of timely coding a depth map that predicts the coding units (CUs) division for advance 3D extension of high efficiency video coding (3D-HEVC) is tackled by $\mathrm{H}$. Zhang et al. The authors are proposing to employ a CNN that reduces the coding time by an average of $34.2 \%$, with negligible quality degradation of the synthesized virtual view.

All these papers show that a deep neural network can be applied to multiple fields of image and video compression to replace human crafted algorithms and yield improved compression results.

Ofer Hadar received BSc, MSc (cum laude), and PhD degrees from Ben-Gurion University of the Negev, Israel, in 1990, 1992, and 1997, respectively, all in electrical and computer engineering. In 1999, he joined the Communication Systems Engineering Department at Ben-Gurion University of the Negev. Currently, he is a full professor and was the head of the department between 2012-2018. His current research interests include image and video compression, image and video analytics algorithms using deep learning for various applications, such as, autonomous cars, tele-medicine, remote sensing, precision agriculture, and more. He also works as a consultant of various high-tech companies in Israel and is a senior member of IEEE and SPIE.

Touradj Ebrahimi is professor of image processing at Ecole Polytechnique Fédérale de Lausanne (EPFL) active in teaching and research in multimedia signal processing. He is the

(ㄱ) 2021 SPIE and IS\&T 
head of the Multimedia Signal Processing Group at EPFL. Since 2014, he has been the convener of the JPEG standardization committee, which has produced a family of standards that have revolutionized the world of imaging. He represents Switzerland as the head of its delegation to JTC1 (in charge of standardization of information technology in ISO and IEC), SC29 (the body overseeing MPEG and JPEG standardization) and is a member of ITU representing EPFL. His areas of interest include image and video compression, media security, quality of experience in multimedia and AI based image and video processing and analysis. He is a fellow of the IEEE and SPIE and has been recipient of several awards and distinctions, including an IEEE Star Innovator Award in Multimedia. 